\title{
Review
}

\section{Automated Systems Based on Wearable Sensors for the Management of Parkinson's Disease at Home: A Systematic Review}

\author{
AU1 Erika Rovini, MD, ${ }^{1}$ Carlo Maremmani, $P h D^{2}$ \\ and Filippo Cavallo, $\mathrm{PhD}^{1}$ \\ ${ }^{1}$ The BioRobotics Institute, Scuola Superiore Sant'Anna, \\ Pontedera (PI), Italy. \\ ${ }^{2}$ U.O. Neurologia, Ospedale delle Apuane (AUSL Toscana \\ Nord Ovest), Massa (MS), Italy.
}

\begin{abstract}
Background: Parkinson's disease is a common neurodegenerative pathology that significantly influences quality of life (QoL) of people affected. The increasing interest and development in telemedicine services and internet of things technologies aim to implement automated smart systems for remote assistance of patients. The wide variability of Parkinson's disease in the clinical expression, as well as in the symptom progression, seems to address the patients' care toward a personalized therapy.

Objectives: This review addresses automated systems based on wearable/portable devices for the remote treatment and management of Parkinson's disease. The idea is to obtain an overview of the telehealth and automated systems currently developed to address the impairments due to the pathology to allow clinicians to improve the quality of care for Parkinson's disease with benefits for patients in QoL.

Data Sources: The research was conducted within three databases: IEEE Xplore ${ }^{\circledR}$, Web of Science ${ }^{\circledR}$, and PubMed Central $^{\circledR}$, between January 2008 and September 2017.

Study Eligibility Criteria: Accurate exclusion criteria and selection strategy were applied to screen the 173 articles found. Results: Ultimately, 55 articles were fully evaluated and included in this review. Divided into three categories, they were automated systems actually tested at home, implemented mobile applications for Parkinson's disease assessment, or described a telehealth system architecture.

Conclusion: This review would provide an exhaustive overview of wearable systems for the remote management and automated assessment of Parkinson's disease, taking into account the reliability and acceptability of the implemented technologies.
\end{abstract}

Keywords: eHealth, IoT, m-Health, Parkinson's Disease, telemedicine, telemonitoring, wearable sensors

\section{Introduction}

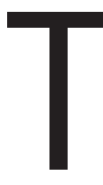
he increasing aging of population is resulting in a wide prevalence of chronic diseases such as neurodegenerative disorders, which require long-term high-cost treatments. ${ }^{1}$ A plan to develop strategies able to reduce healthcare costs for age-related pathologies is an important challenge for the future. ${ }^{2,3}$ In this scenario, Parkinson's disease (PD) is a neurodegenerative illness that affects millions of people worldwide, and its incidence is growing. ${ }^{4}$ PD is caused by a critical loss of dopamine in the forebrain, which results in typical cardinal motor symptoms such as tremor, postural instability, muscular rigidity, and bradykinesia/hypokinesia. ${ }^{5}$ In addition, common nonmotor symptoms such as autonomic dysfunctions, sleep disturbances, and olfactory symptoms are critical factors that degrade the quality of life (QoL) of PD patients ${ }^{6}$ and make the pathology severely disabling, both physically and socially. ${ }^{7,8}$

The long-term development of the disease, expression of symptomatic complications (e.g., motor fluctuations) during specific times of day, long waiting lists, and traveling costs (particularly for people who live in rural areas) are just a few reasons that support the need to move Parkinson's care into the home and to develop new care models. ${ }^{9}$

The wide variability in clinical expression, as well as in progression of somatic symptoms, ${ }^{10}$ makes the pathology difficult to adequately identify and treat. ${ }^{11}$ Particularly, as the pathology onset appears unilaterally, with specific impairments, and it develops differently among patients, ${ }^{12}$ it makes sense that a personalized and adapted therapy should be administered based on the individual needs of PD patients. This approach would enable optimal care and would treat the predominant symptoms. ${ }^{13}$ The approach would also meet the principles of the novel precision medicine concept for healthcare systems, ${ }^{14}$ which aim to provide the best available care for each subject. In particular, precision medicine is expected to integrate the best evidence-based knowledge in different fields, including molecular imaging, deep brain 


\section{ROVINI ET AL.}

stimulation, and wearable sensors, to identify the optimal solution for the treatment and management of PD patients. ${ }^{15}$ Personalized strategies are also important to support patients' engagement in their care path, making them active in their own health. ${ }^{16}$

Recently, advances in wearable sensors, information, and communication technologies, as well as data mining, are promoting the design and the implementation of e-Health systems that allow to provide novel therapeutic and monitoring solutions for PD patients. ${ }^{17}$ These systems aim to maximize the efficiency of healthcare, enhancing its quality without increasing costs. This is accomplished through augmented contacts between patients and clinicians and sharing information between the different stakeholders. These systems also promote the empowerment of patients to actively manage their health and to adopt healthy behaviors. ${ }^{18}$ In the same vein, internet of things (IoT) systems for healthcare are emerging. ${ }^{19}$ They can allow the collection of huge amounts of patients' data through wearable sensors that are connected to a medical database through mobile devices. These data are analyzed by intelligent algorithms to obtain useful information for discriminating relevant health conditions, adjusting therapy, monitoring disease progression, and supporting both clinicians and patients in decision-making.

This review article aims to provide a wide overview of the telehealth and automated systems currently developed to address the impairments caused by PD to allow the clinicians to improve the quality of care for the pathology and provide relative benefits for patients in QoL. In particular, this article provides a review of the typologies of smart systems that were investigated and implemented for PD management in the last decade and focuses both on the kind of technology used and the system performance. Such systems are organized into three different categories on the basis of their level of development. Indeed, some works presented technologies able to provide automatic assessment of one or more specific impairments in $\mathrm{PD}$, and they were tested in the actual home environment. Alternatively, other studies focused on the development of mobile health applications for remote automated PD management. Finally, several articles described the architecture of a telemedicine system for the provision of a remote healthcare service for PD patients. For each category, the existing systems that emerged from the review process were investigated, analyzed, and discussed.

\section{Materials and Methods}

\section{DATA SOURCES}

An electronic database search was performed on September 15, 2017 using IEEE Xplore ${ }^{\circledR}$, Web of Science ${ }^{\circledR}$, and PubMed
Central $^{\circledR}$ to identify articles concerning the use of wearable sensors for automated and remote management of Parkinson's disease. According to the PRISMA statement, ${ }^{20}$ an additional manual search was performed (e.g., through citations of articles included in this review), but no further articles were relevant for inclusion in this review article.

\section{SEARCH TERMS}

Specifically, the terms and keywords used for the literature research were ("Parkinson") AND ("wearable" OR "inertial" OR “accelerometer" OR "acceleration" OR "gyroscope” OR “EMG” OR “EEG” OR “ECG” OR “GSR” OR “clothes”) AND ("Telemedicine" OR "Telehealth" OR "Telecare" OR "Telemonitoring" OR "mhealth" OR "ehealth" OR "M-Health" Or "E-Health" OR “Mobile Health" OR "Home Monitoring” OR "IoT" OR "Internet of Things") located within title and/or abstract and/or keywords.

\section{STUDY SELECTION PROCESS}

Only original full-text articles published in English, between January 2008 and September 2017, which discussed the use of wearable/portable sensors for automated remote PD assessment and management, were included in this review. First, duplicated references were manually identified and excluded. Then, during the screening procedure, items were excluded if: (1) they were an abstract, a letter, a review article, or a chapter from a book or (2) they were not written in the English language. Each author independently screened the articles that were excluded with reason if: (1) they did not use any type of wearable/portable sensors; (2) they did not manage Parkinson's Disease; (3) they did not appear appropriate for this review after the reading of title and abstract; or (4) they were not full access. In addition, (5) if multiple articles written by the same author had similar content, articles published in journals were selected instead of articles presented at conferences. Furthermore, (6) if multiple articles written by the same author with similar content were presented at conferences, the most recent article was selected. Disagreements about the inclusion/exclusion and classification of the articles were solved through meetings and discussions among the authors.

Finally, the selected articles, fully evaluated and included in this review, were classified into three groups based on whether (1) they implemented devices for automatic assessment of PD symptoms or impairments (e.g., freezing of gait [FOG], tremor) and they were tested in the actual home environment; (2) they developed $\mathrm{m}$-health applications on smartphone/tablet for PD; or (3) they designed a telemedicine service, describing its architecture. 
TMJ-2018-0035-ver9-Rovini_1P.3d 06/14/18 3:19pm Page 3

WEARABLE SENSORS FOR PARKINSON'S DISEASE AT HOME

\section{DATA ABSTRACTION}

Data were abstracted from each selected article, as reported in T1 - T3Tables 1-3. For the first 2 categories evidenced the technological solutions used, the experimental aspects, and the performed analysis. In particular, for the technology, the typology of the sensors, their placement over the body, and the sampling frequency were reported. About the experimental sessions, the designed protocol adopted and the subjects involved according to their pathology and their health status were described. Furthermore, the last three columns of the Tables 1-3 synthetized the extracted features, the applied statistical methods, the implemented classifiers, and the main findings for each work. Differently, for the third category, the focus was on the technology used, the symptom addressed, the architecture and performance of the telehealth system, and the preventative measures adopted in terms of privacy and secure transmission of data.

\section{Results}

\section{APPLICATION OVERVIEW}

Obtained in the research were 173 articles: 62 references were retrieved from IEEE Xplore, 43 references were obtained from Web of Science, and 68 references were taken from PubMed Central. After removing the duplicated items, 106 references were fully assessed within the evaluation procedure. Finally, according to the eligibility criteria, 55 articles resulted, which were adequate for the present work and were included in the final review (Fig. 1).

\section{ANALYSIS METHODS}

The selected articles for this review were divided into three categories based on their level of development. The first category included works that implemented technological solutions for the automatic assessment of one or more specific symptoms of PD. The designed protocol for these studies demanded that experimental sessions be performed in the home environment. The automated evaluation of PD impairments represents the first step toward a telemedicine system and allows the patients to automatically monitor their health status; this application is particularly valuable for the most disabling symptoms. The second category of articles encompassed studies in which a mobile application was developed for PD management. This category is a further step toward the fully automated assessment of the pathology at home, allowing the patients to use a highly common technological device (i.e., the smartphone) that could acquire data directly, process the data, and eventually provide timely feedback to the users. Finally, the third category featured researches in which telehealth services were designed and/or implemented. This class can include works from other categories, such as wearable solutions tested in the home environment or mobile apps for PD. In addition, the class can provide the complete system architecture, which integrates wearable sensors, mobile/web-based applications, servers for the storage and elaboration of acquired data, and smart interfaces for communication between patients, caregivers, and medical staff (Fig. 2).

\section{REPORTED RESULTS}

More than half (52.7\%) of the 55 fully evaluated articles were published during the past 3 years and 74.5\% over the past 5-year period (Fig. 3a). This result confirmed the increasing interest for wearable systems in PD remote applications, as well as the need to improve telecare services for chronic patients. Thirty-one articles (56.4\%) were published in journals, while the others were presented in international conferences. Regarding the application, 30 studies (54.4\%) were tested in the home environment, 21 works (38.2\%) implemented mobile applications, and 24 articles (43.6\%) presented telehealth systems for PD. Nevertheless, only 4 articles (7.2\%) concerned all three topics (Fig. 3b).

\section{AUTOMATED SYSTEMS TESTED AT HOME}

The possibility to have an automated evaluation of specific symptoms or disturbances can represent the first step toward a remote management of the disease. In particular, some impairments such as FOG,${ }^{21-23}$ risk of falls, ${ }^{24}$ motor fluctuations, and dyskinesias ${ }^{25-31}$ can mainly occur during specific times over the day. For this reason, it is not easy for the neurologist to evaluate the symptoms during a clinical examination at the hospital. The opportunity to measure and analyze motor performances at home can allow the patients to record their impairments precisely when they first appear, allowing the clinician to keep track of them over time. Accelerometers (ACC) and gyroscopes (GYR) are the most used sensors for these measurements (Table 1) because they represent a valid trade-off between unobtrusiveness and accuracy for motion measures. ${ }^{30,32-34}$ Furthermore, they can eventually be included in a smartphone, ${ }^{22,35}$ a wristband, ${ }^{29}$ or a smartwatch, ${ }^{36,37}$ which are common technological tools, to increase the acceptability and usability of these systems. ${ }^{35}$ These works aimed to address one or more symptoms, and their feasibility was typically measured by evaluating the ability of the system to discriminate PD patients from healthy subjects of controls ( $\mathrm{HC})^{26,38-45}$ or to correlate well with UPDRS clinical scores. ${ }^{26,30,40,41,46}$ Machine learning approaches (e.g., Support Vector Machine, Decision Tree, Random Forest, and Neural Networks) were generally implemented to evaluate the accuracy of the system in assessing the investigated symptoms. ${ }^{21-23,25,27,28,30,36,38,47}$ However, sometimes long-term studies involved a limited number of 
TMJ-2018-0035-ver9-Rovini_1P.3d 06/14/18 3:19pm Page 4

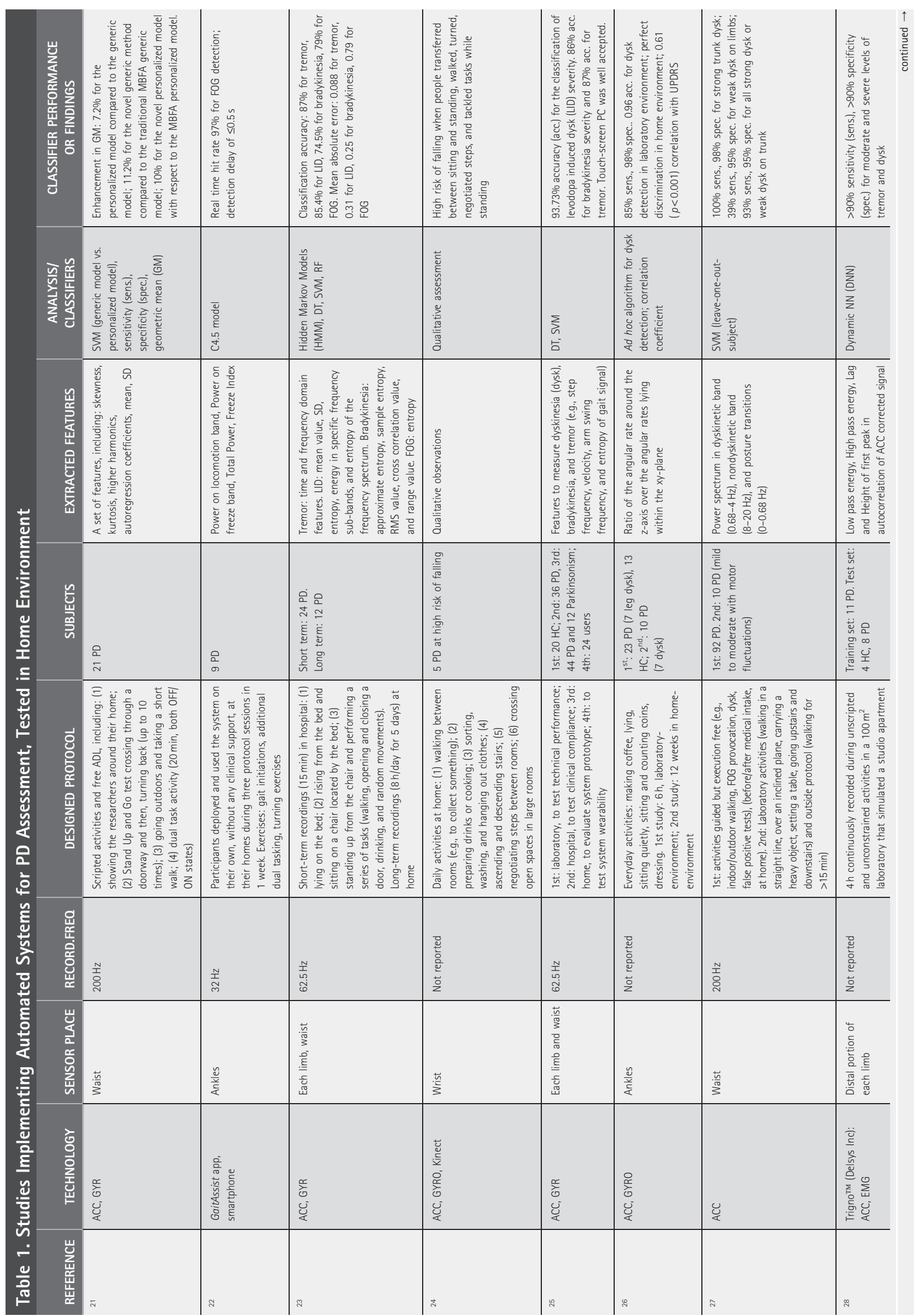


TMJ-2018-0035-ver9-Rovini_1P.3d 06/14/18 3:19pm Page 5

\begin{tabular}{|c|c|c|c|c|c|c|c|c|c|c|}
\hline & 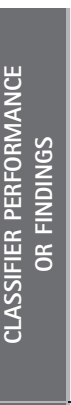 & 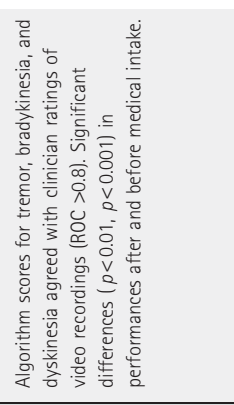 & 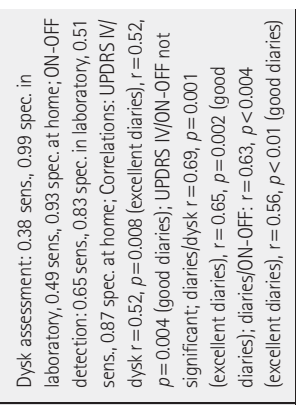 & 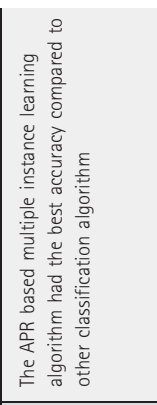 & 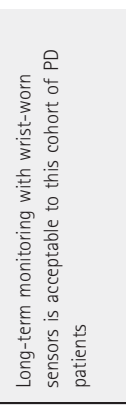 & 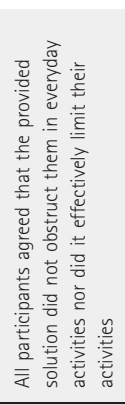 & 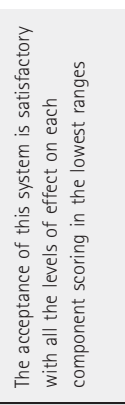 & 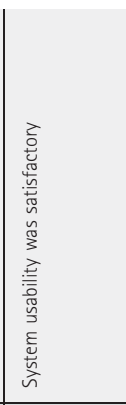 & 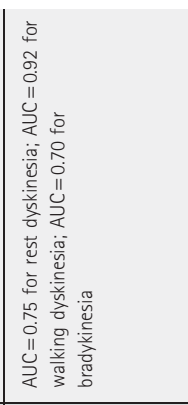 & 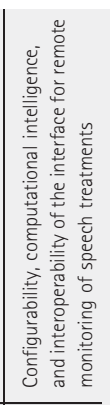 \\
\hline & 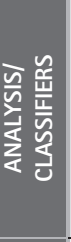 & 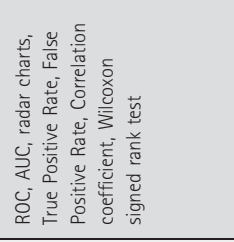 & 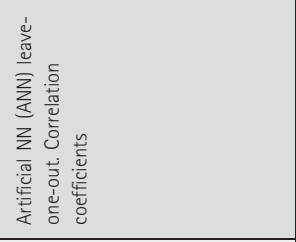 & 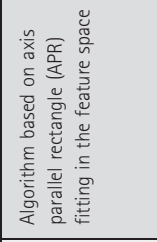 & 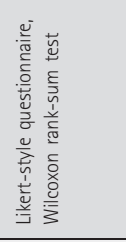 & 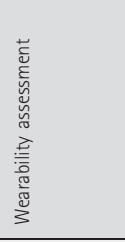 & 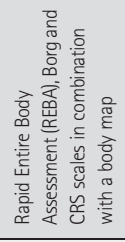 & 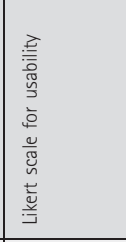 & 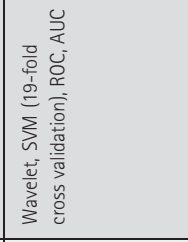 & 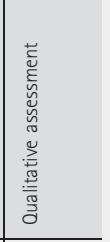 \\
\hline & 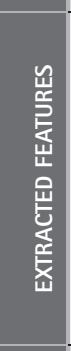 & 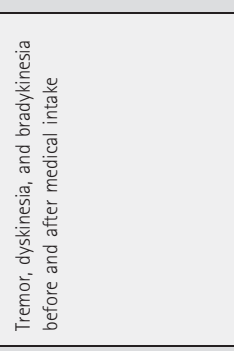 & 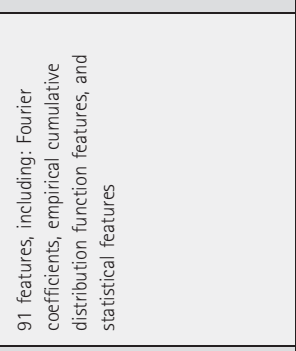 & 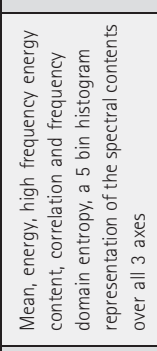 & 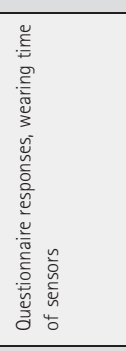 & 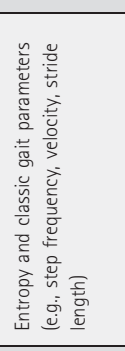 & 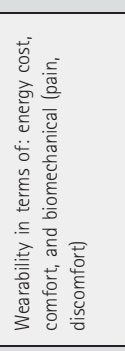 & 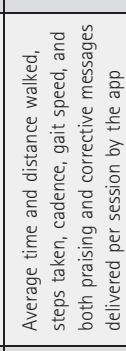 & 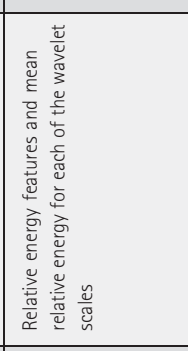 & 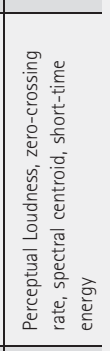 \\
\hline & 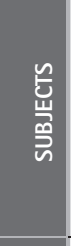 & 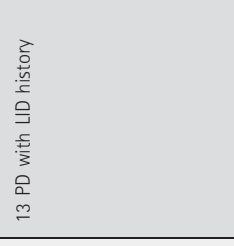 & 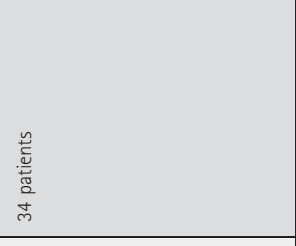 & $\stackrel{a}{a}$ & 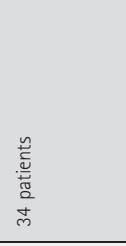 & $\stackrel{\text { a }}{=}$ & $\underset{\substack{a \\
\grave{\infty}}}{a}$ & $\stackrel{a}{a}$ & \begin{tabular}{|l}
0 \\
2 \\
2 \\
\end{tabular} & $\stackrel{a}{\circ}$ \\
\hline 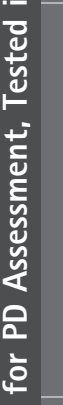 & 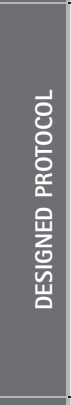 & 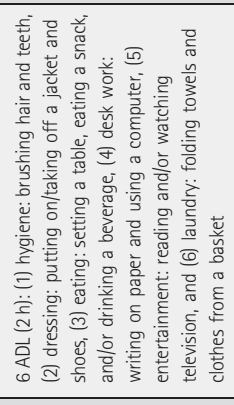 & 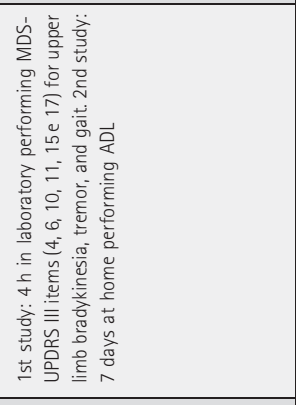 & 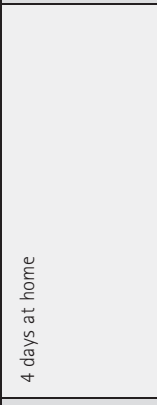 & 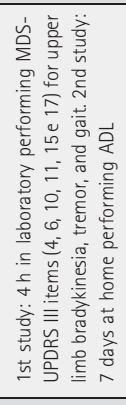 & 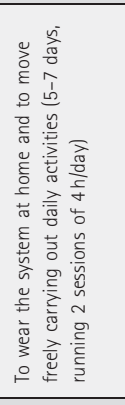 & 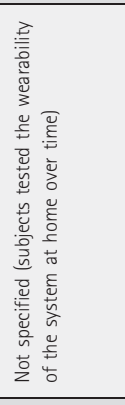 & 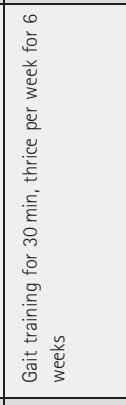 & 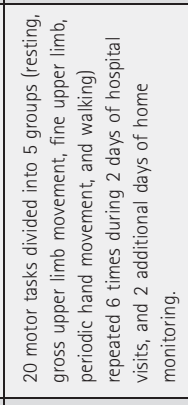 & 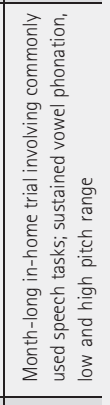 \\
\hline 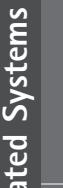 & 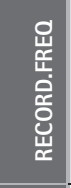 & 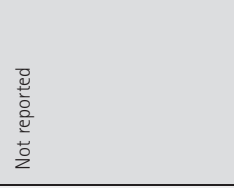 & $\stackrel{\underline{x}}{\underline{8}}$ & 章 & $\begin{array}{l}\underline{N} \\
\stackrel{8}{8}\end{array}$ & 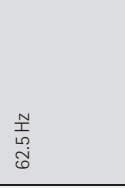 & 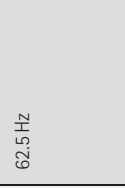 & 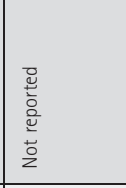 & 営 & 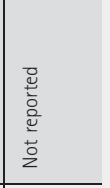 \\
\hline 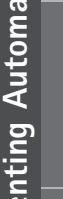 & 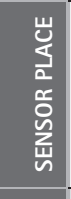 & 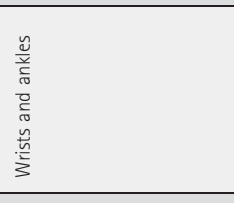 & $\frac{n}{\frac{n}{3}}$ & 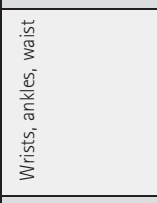 & $\frac{\underline{n}}{\bar{y}}$ & 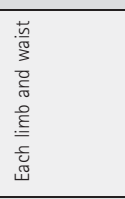 & 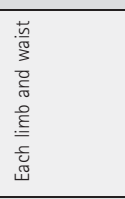 & 总 & 蒿 & 蒿 \\
\hline$\frac{\mathrm{g}}{\mathrm{g}}$ & 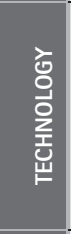 & 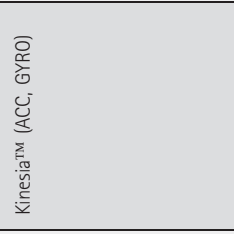 & $\ddot{x}$ & $\ddot{z}$ & $\ddot{z}$ & 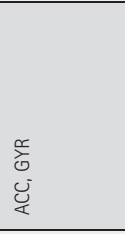 & $\begin{array}{l}\frac{9}{5} \\
\text { y }\end{array}$ & 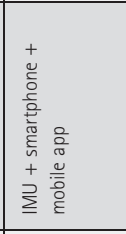 & 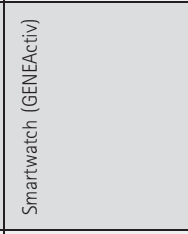 & 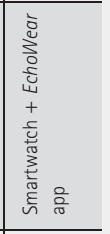 \\
\hline - & 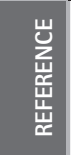 & & $\therefore$ & & $\approx$ & 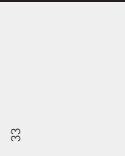 & 声 & $\stackrel{2}{\circ}$ & 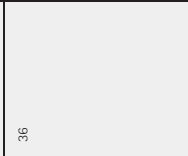 & $\therefore$ \\
\hline
\end{tabular}


TMJ-2018-0035-ver9-Rovini_1P.3d 06/14/18 3:19pm Page 6

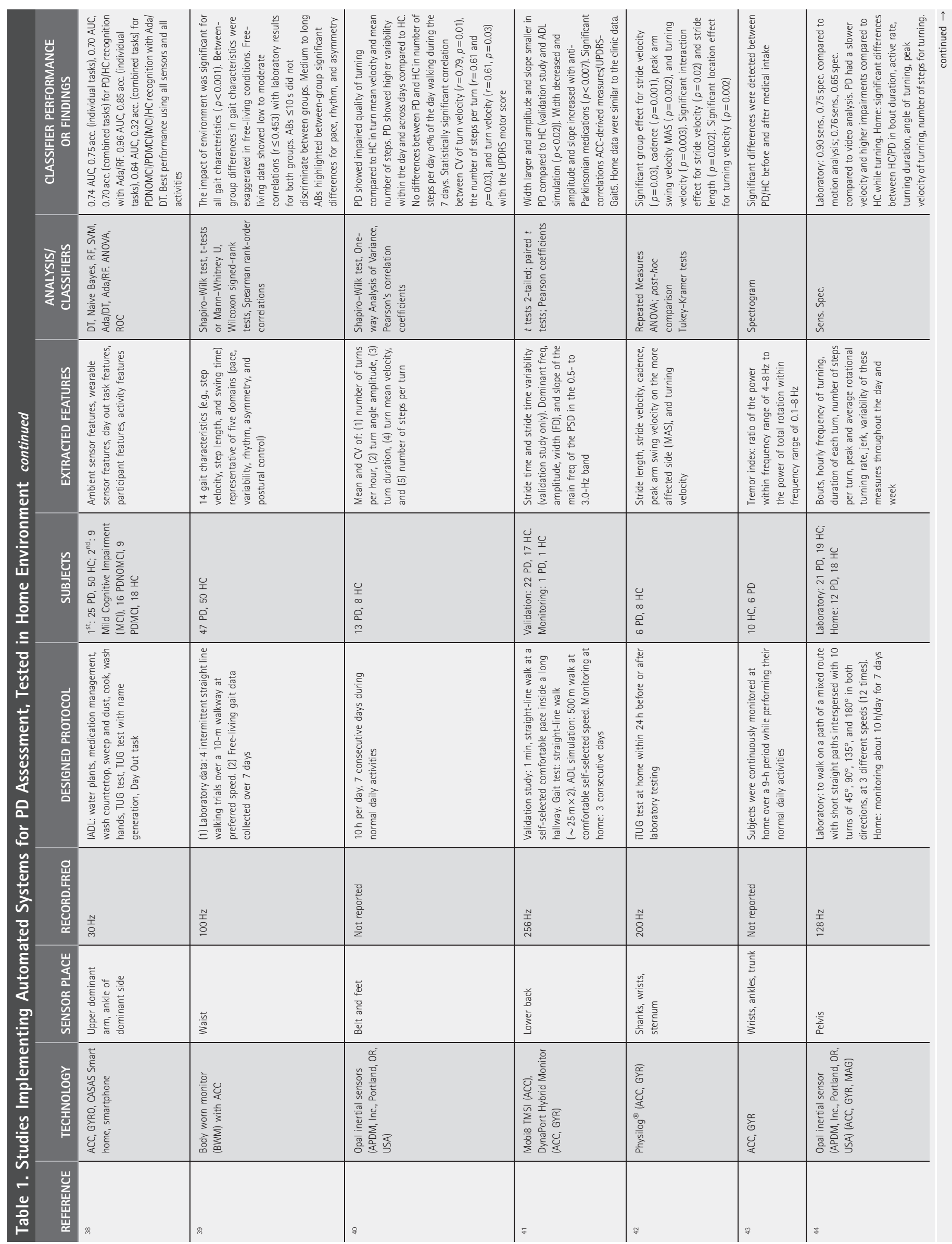


TMJ-2018-0035-ver9-Rovini_1P.3d 06/14/18 3:19pm Page 7

\begin{tabular}{|c|c|c|c|c|c|c|}
\hline 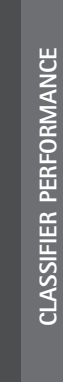 & 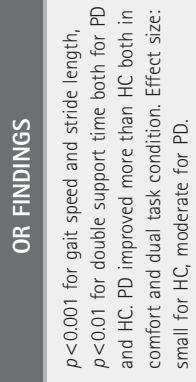 & 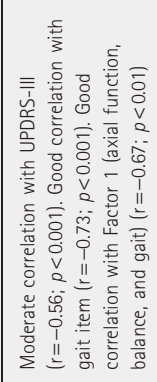 & 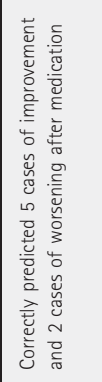 & 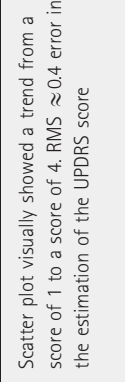 & 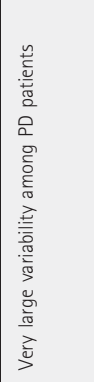 & 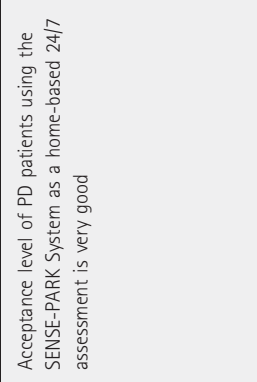 \\
\hline & 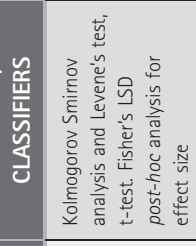 & 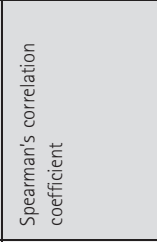 & 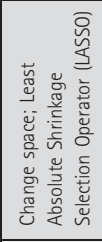 & 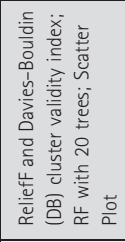 & 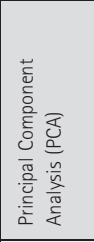 & 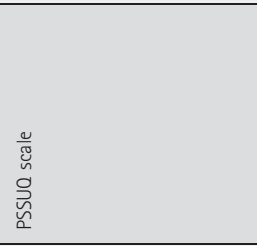 \\
\hline & 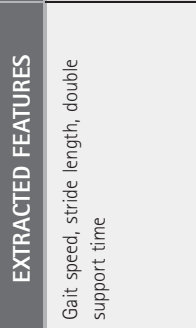 & 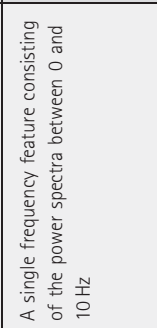 & 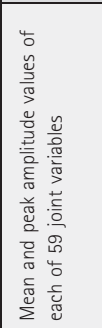 & 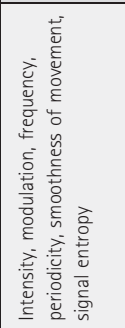 & 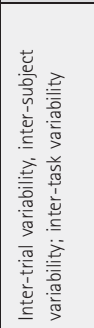 & 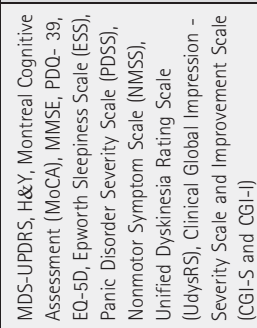 \\
\hline & 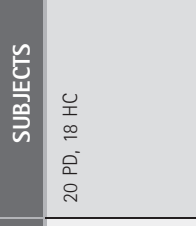 & 高 & $\stackrel{ }{\stackrel{2}{ }}$ & $\begin{array}{l}a \\
\text { a } \\
w 0\end{array}$ & $\stackrel{\circ}{\stackrel{2}{=}}$ & $\begin{array}{l}\text { a } \\
\text { in }\end{array}$ \\
\hline & 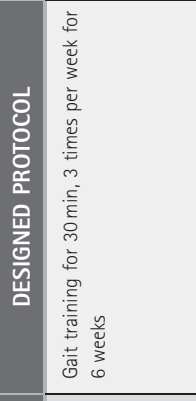 & 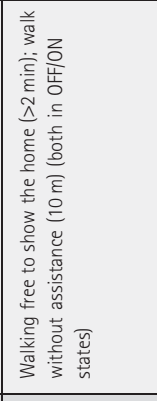 & 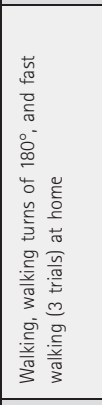 & 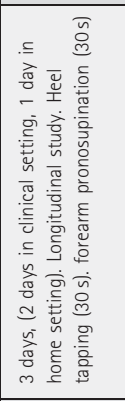 & 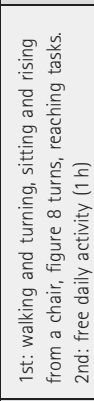 & 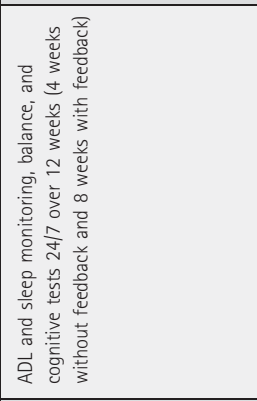 \\
\hline & 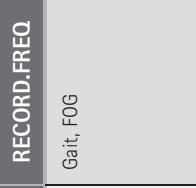 & 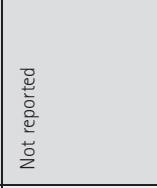 & $\stackrel{\underline{N}}{\underline{0}}$ & 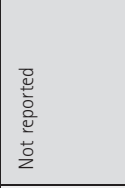 & \begin{tabular}{|l} 
No \\
$\stackrel{\underline{0}}{0}$
\end{tabular} & 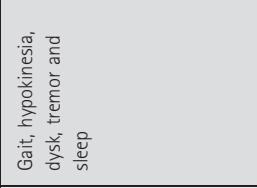 \\
\hline & 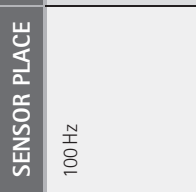 & $\frac{\frac{\hbar n}{m}}{3}$ & 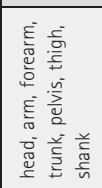 & 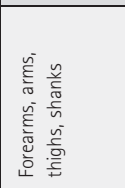 & 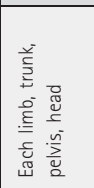 & 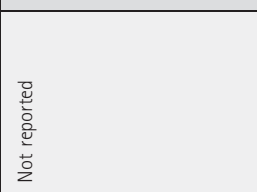 \\
\hline & 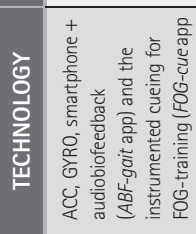 & 安 & 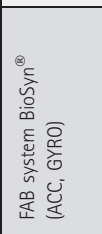 & 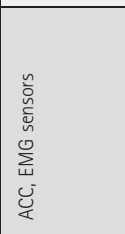 & 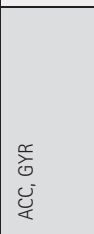 & 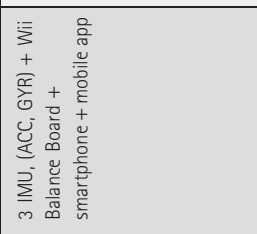 \\
\hline & 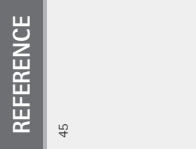 & 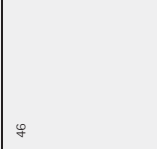 & $\Rightarrow$ & q & F & $\therefore$ \\
\hline
\end{tabular}




\begin{tabular}{|c|c|c|c|c|c|c|c|}
\hline & 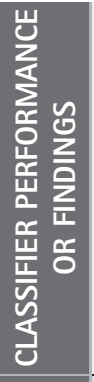 & 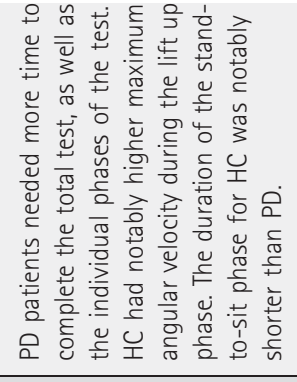 & 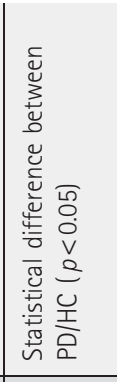 & 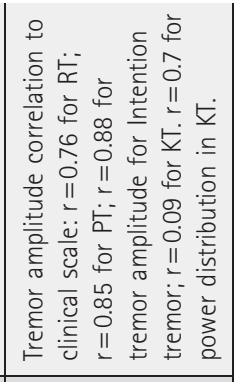 & 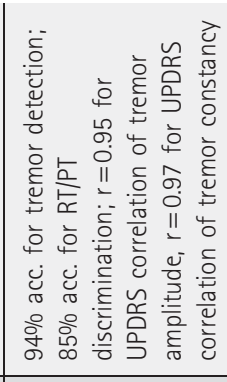 & 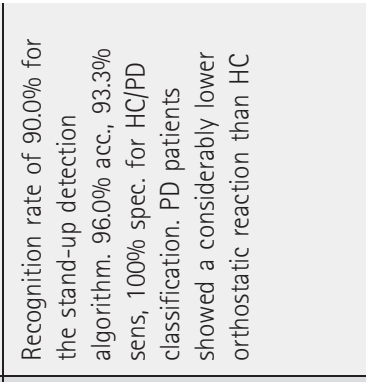 & 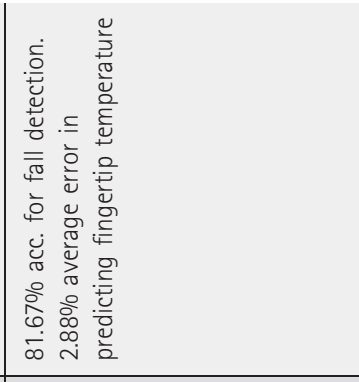 \\
\hline & 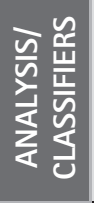 & 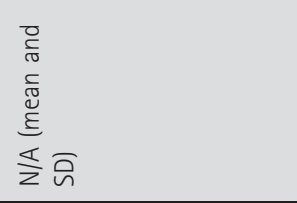 & 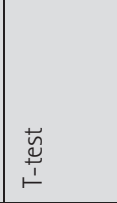 & 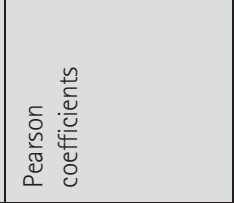 & 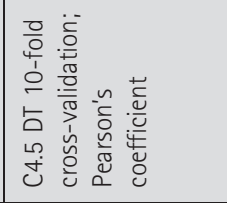 & 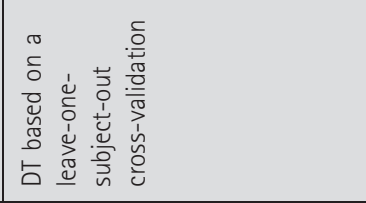 & \begin{tabular}{|l}
$\frac{0}{3}$ \\
$\frac{2}{3}$ \\
$\frac{N}{3}$ \\
$\frac{3}{3}$
\end{tabular} \\
\hline & 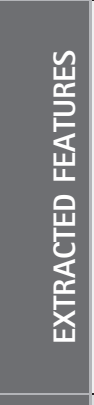 & 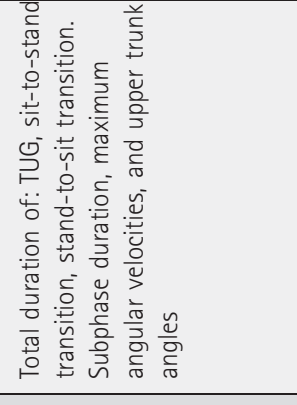 & 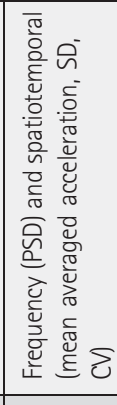 & 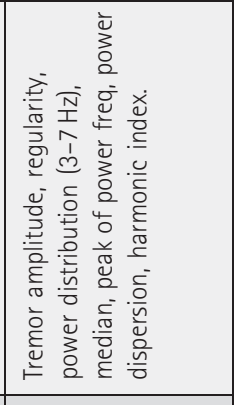 & 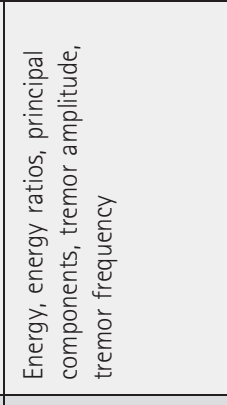 & 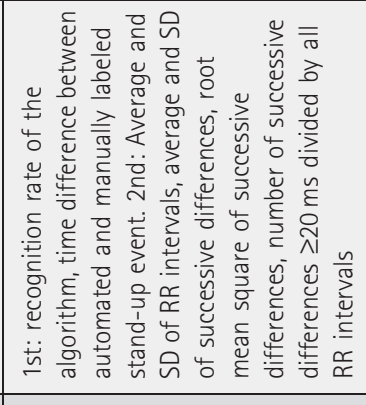 & 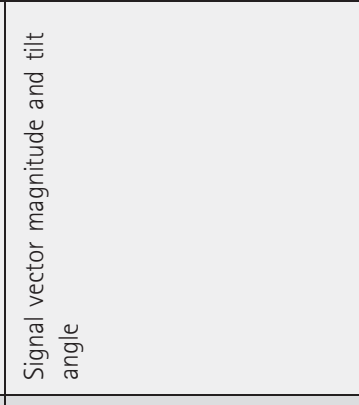 \\
\hline & 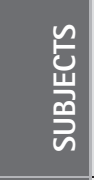 & $\begin{array}{l}y \\
0 \\
x \\
\vdots \\
\vdots \\
m \\
m\end{array}$ & $\begin{array}{l}\text { ỹ } \\
\bar{a} \\
\text { à } \\
-\end{array}$ & 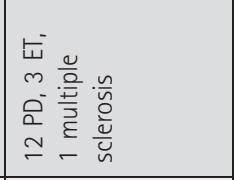 & 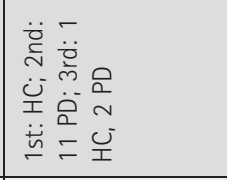 & 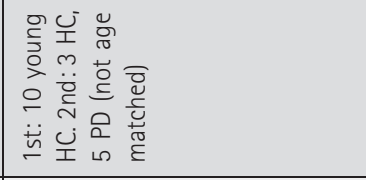 & 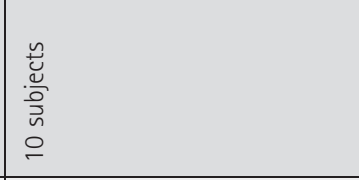 \\
\hline & 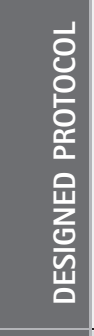 & 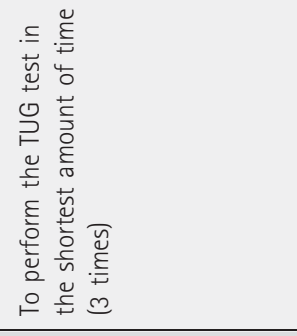 & 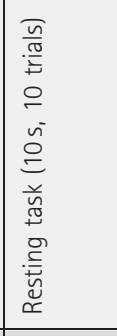 & 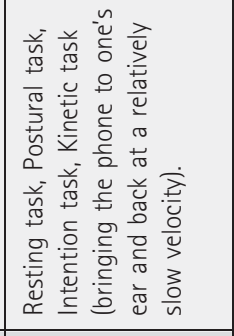 & 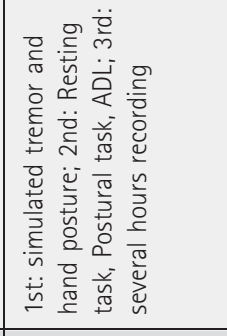 & 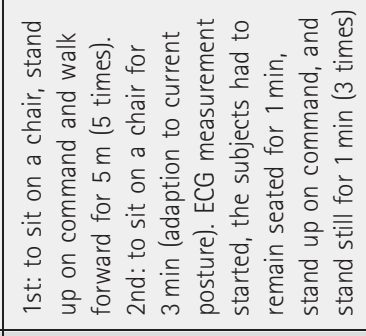 & 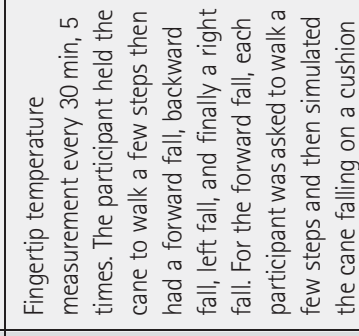 \\
\hline 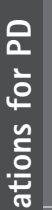 & 竞 & 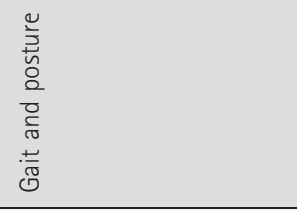 & 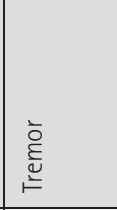 & 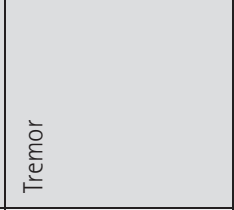 & 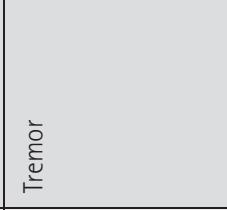 & 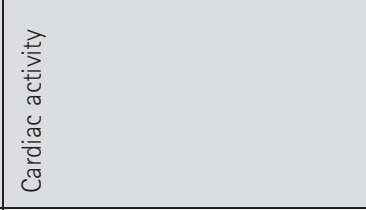 & \begin{tabular}{|l}
$\frac{\varrho}{\bar{~}}$ \\
\end{tabular} \\
\hline & 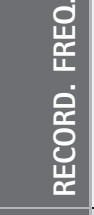 & $\begin{array}{l}\text { Nㅗㅇ } \\
\stackrel{9}{\subseteq} \\
\end{array}$ & 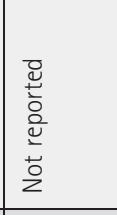 & 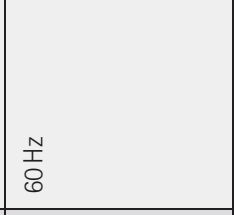 & 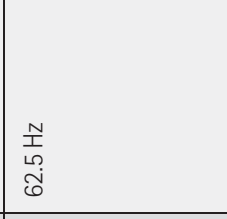 & \begin{tabular}{|l} 
N \\
co \\
$\stackrel{N}{N}$ \\
\end{tabular} & 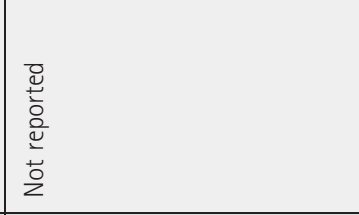 \\
\hline 害 & 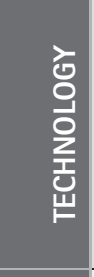 & 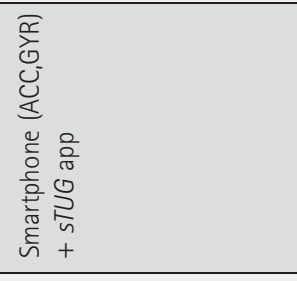 & 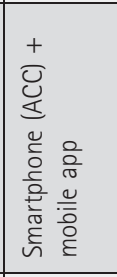 & 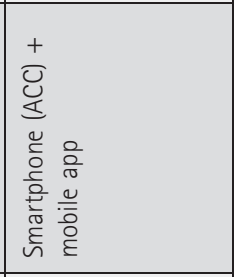 & 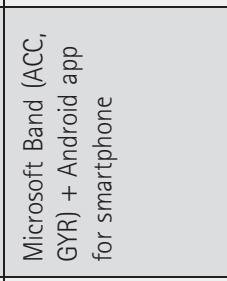 & 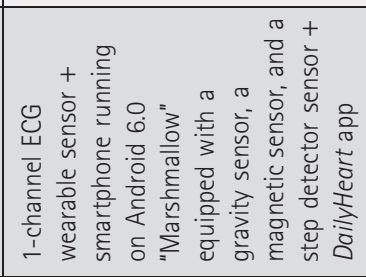 & 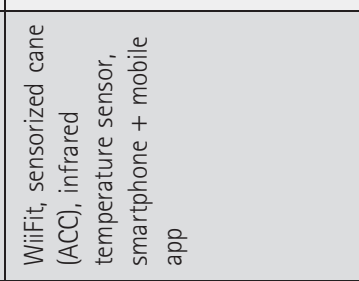 \\
\hline$\frac{c}{d}$ & 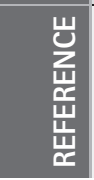 & & in & 古 & 吕 & $\stackrel{\leftrightarrow}{\circ}$ & in \\
\hline
\end{tabular}


TMJ-2018-0035-ver9-Rovini_1P.3d 06/14/18 3:19pm Page 9

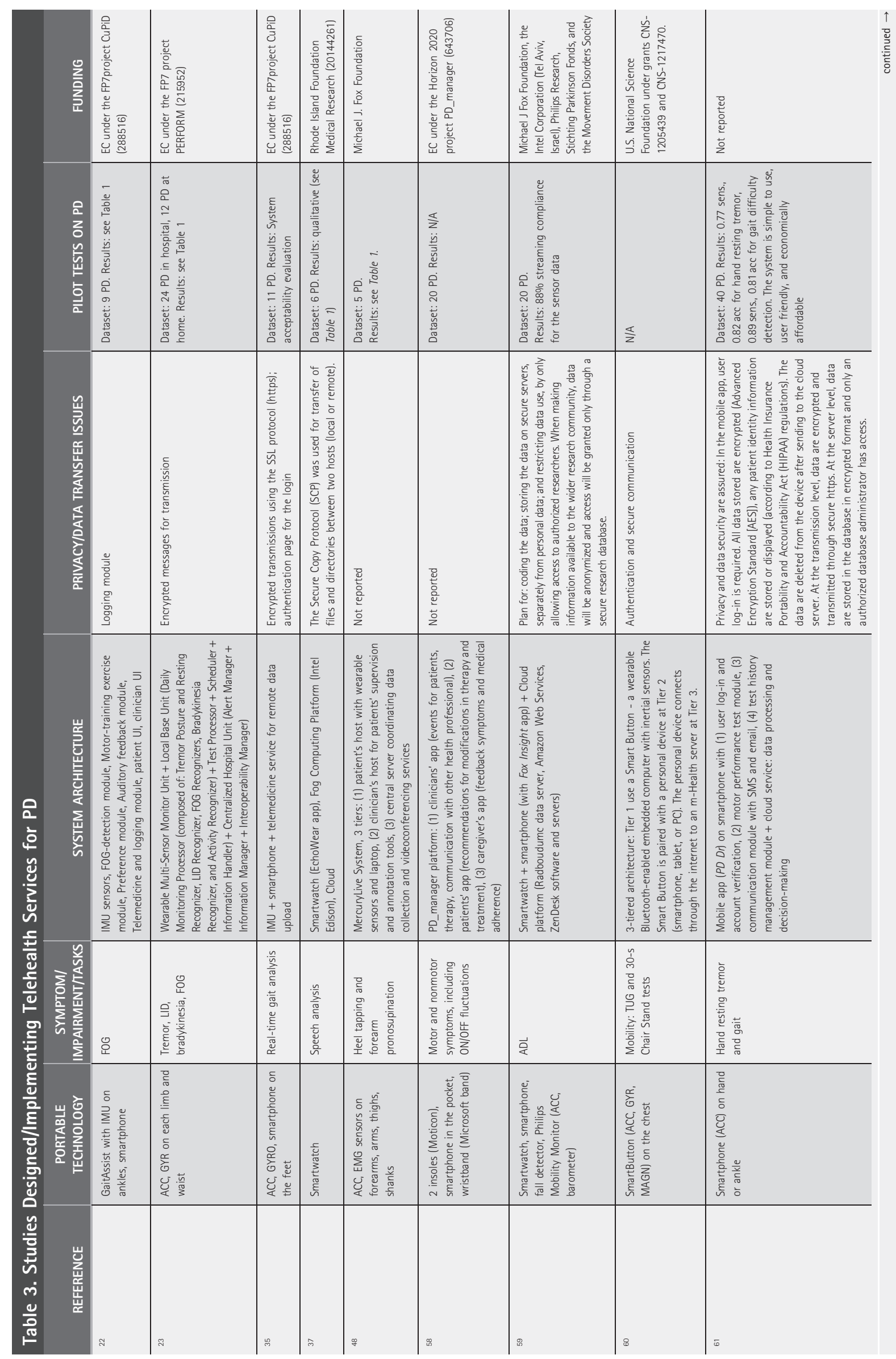


TMJ-2018-0035-ver9-Rovini_1P.3d 06/14/18 3:19pm Page 10

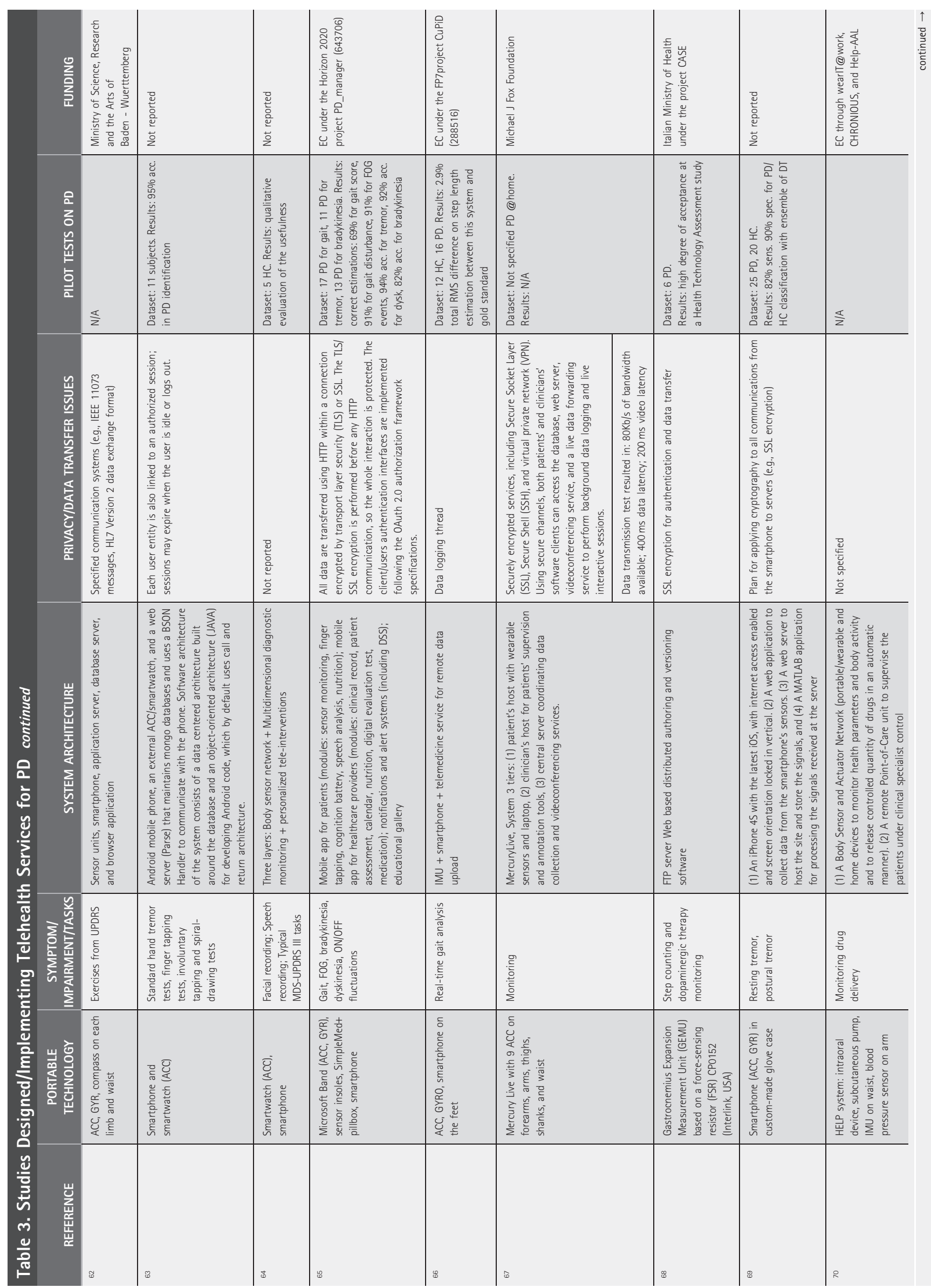


TMJ-2018-0035-ver9-Rovini_1P.3d 06/14/18 3:19pm Page 11

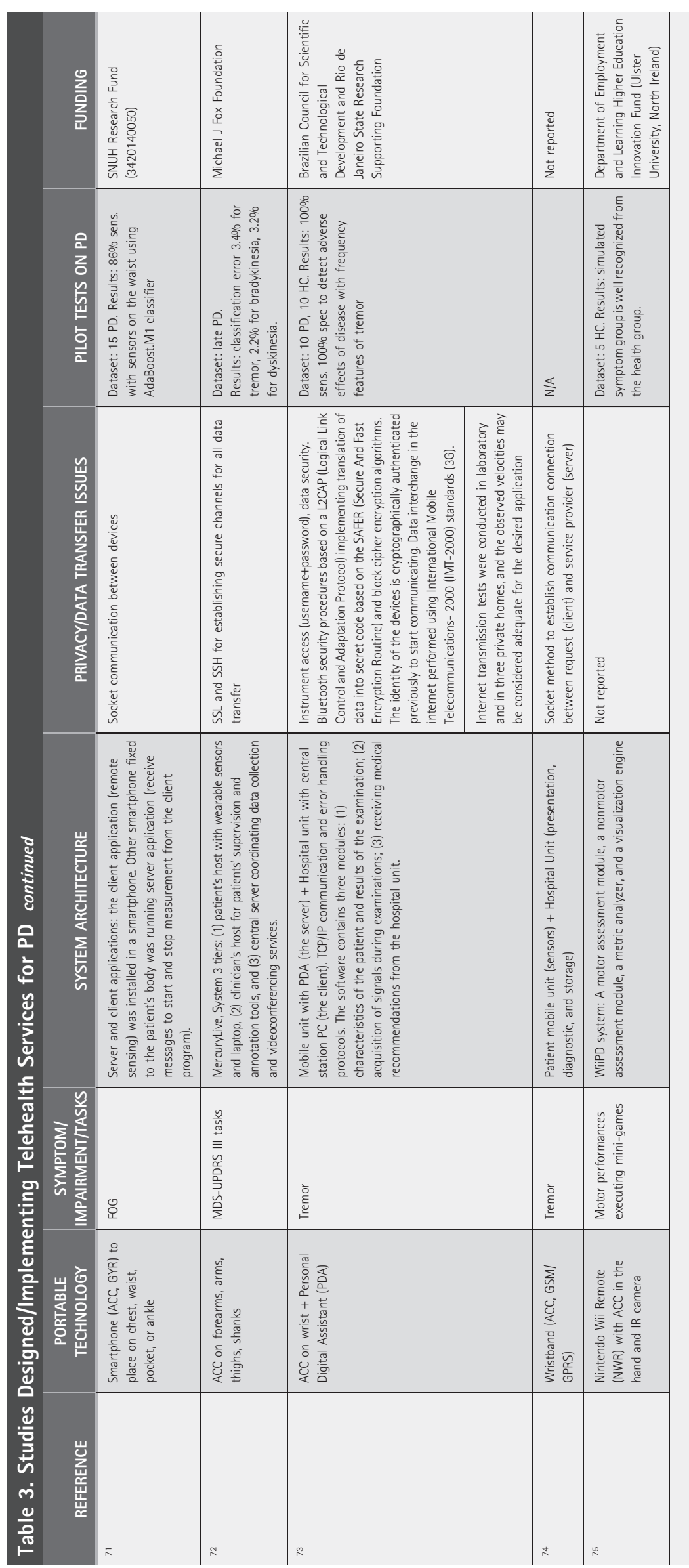

(c) MARY ANN LIEBERT, INC. • VOL. oo NO. oo • MONTH 2018 TELEMEDICINE and e-HEALTH 11 


\section{ROVINI ET AL.}

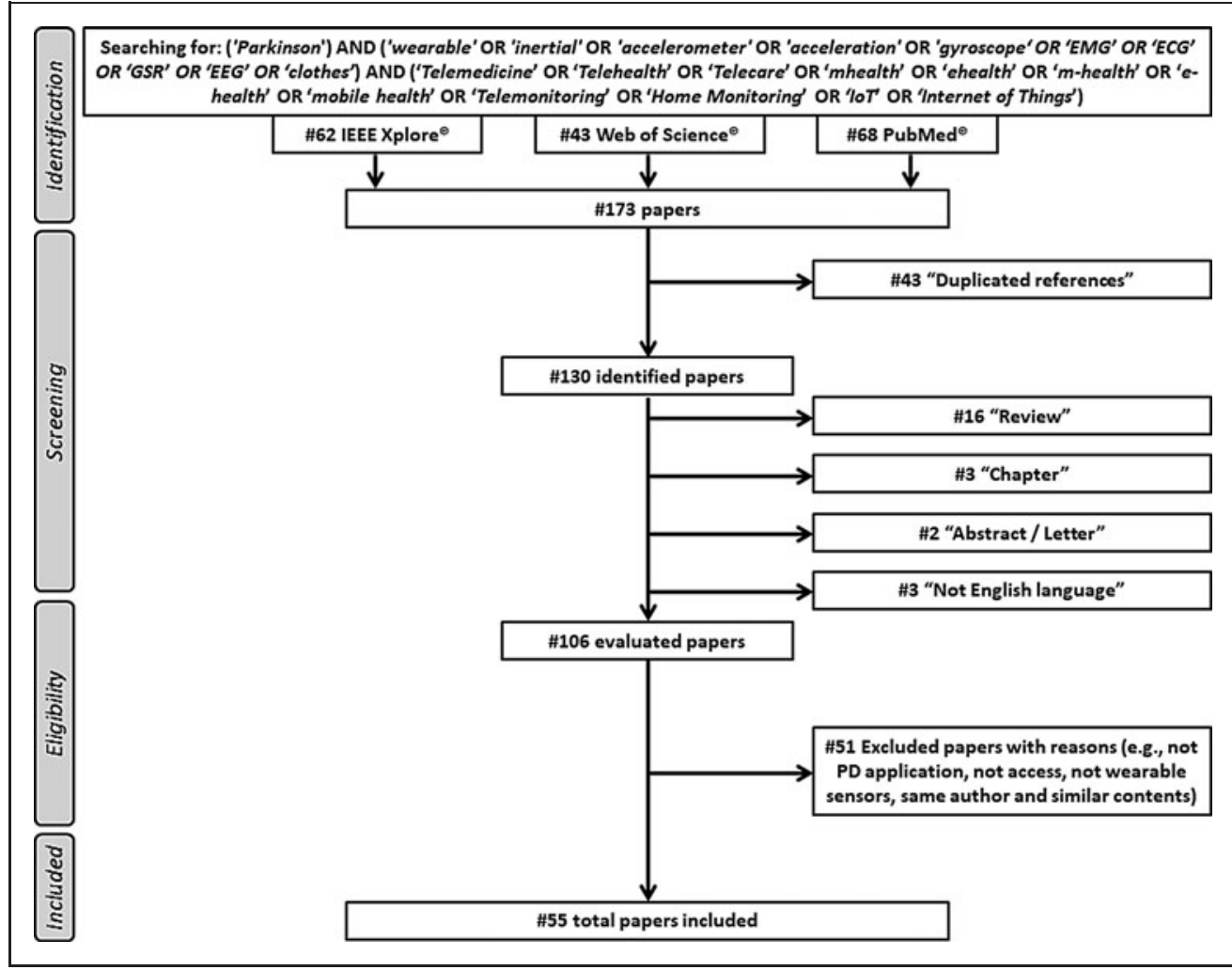

Fig. 1. Selection flowchart according to PRISMA statement. of applications developed and downloaded. ${ }^{51}$ The idea to design mobile apps for monitoring and assessing one or more symptoms in PD reflects the patients' needs to have an objective support for health monitoring during their daily activities without impact on them, both physically and socially. In Table 2 are reported only the works in which a mobile app was developed; m-apps tested at home are already reported in Table $1,{ }^{22,23,35,37,45,50}$ and articles that included m-apps in a complete telemedicine system are reported in Table 3. The mobile apps can be used directly on the patient's own smartphone or smartwatch, without additional devices, minimizing their obtrusiveness, ${ }^{22,37,52-54}$ or they can be integrated with other sensors to improve the measurement subjects, so classification approaches were unreliable, and the statistical analysis was limited to significance and correlation of the features, ${ }^{29,39-42,45,46}$ or it was conducted only visually ${ }^{43,48,49}$ or qualitatively. ${ }^{24,32-35,37,50}$ In Table 1 are reported all the articles that performed tests at home (or simulated home $\mathrm{e}^{28,29}$ ), including systems implementing also mobile apps, ${ }^{45,50}$ a Web-based telemedicine system, ${ }^{48}$ and the combination of all these topics. ${ }^{22,23,35,37}$

\section{MOBILE APPS FOR PD}

In agreement with the large use of smartphones in the population, mobile applications (m-apps) are commonly used in many fields (e.g., gaming and fitness). Healthcare, in particular, is one of the most typical fields for number

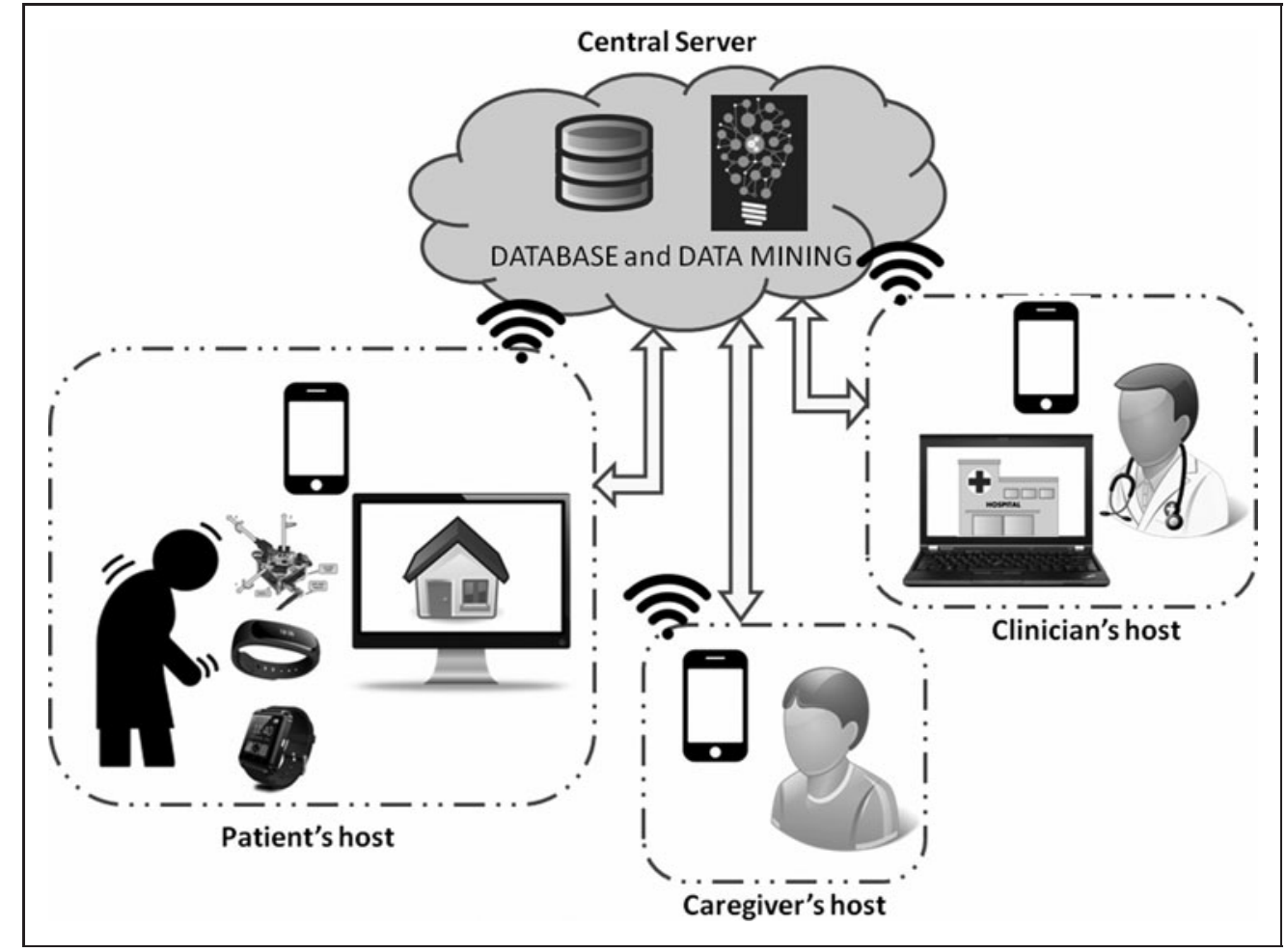

Fig. 2. The architecture of a general telehealth system. 


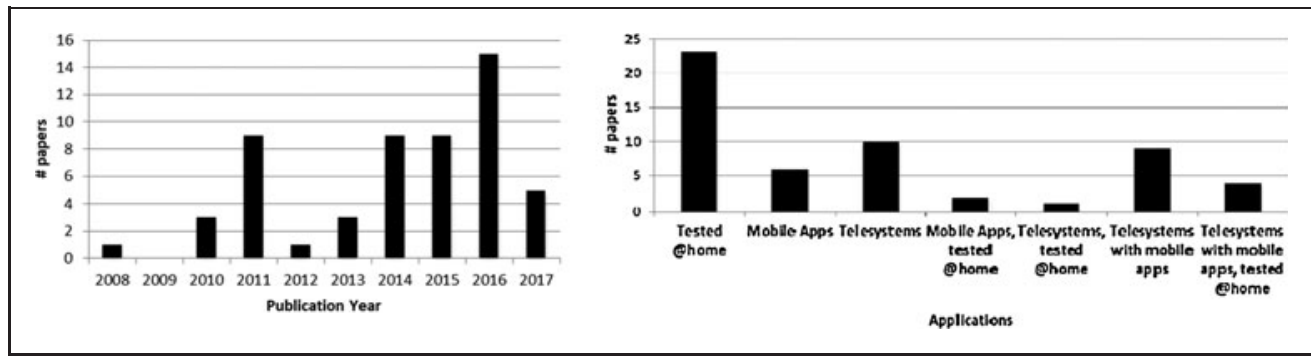

AU7

Fig. 3. Publication trend per year (a); paper distribution per application (b).

transmission and privacy represent important issues to address by adopting preventative measures for data protection. . $^{23,35,37,60,61,63,65,67,68,71-74}$

\section{Discussion}

The increasing rate in agerelated pathologies such as Parkinson's disease is causing an increase of chronic pa-

capability. ${ }^{23,35,55-57}$ They can allow the recording of specific exercises (e.g., tremor analysis and TUG test), ${ }^{52-54}$ as well as monitoring and recording of patients over several hours. ${ }^{23,35,50,55}$ The apps can offer corrective feedback as well, encouraging the patients, for instance, to improve their physical activity or medical adherence. ${ }^{35}$

\section{TELEHEALTH SYSTEMS}

The implementation of e-Health or IoT systems is currently the final goal to have a valid solution for PD remote management (Table 3). Such systems included automated systems for the assessment and/or monitoring of specific symptoms, ${ }^{22,23,35,37,48}$ and they developed also mobile ${ }^{58-66}$ or Web-based ${ }^{48,67-69}$ applications that allow both the patients and the clinicians to easily access the system through appropriate user interfaces. These systems would practically provide a modern telemedicine service using cloud platforms and server applications in which smart algorithms are implemented to analyze the acquired data (Fig. 2). Such systems allow a large amount of data to be transferred and managed, providing both the clinicians and the patients with useful information about disease progression and health conditions. Long-term monitoring at home, ${ }^{67}$ eventually during activities of daily living (ADL), ${ }^{59}$ can be useful for the management of both early-mild patients by permitting the evaluation of the response to drug delivery ${ }^{68,70}$ and for mid-advanced patients in which severe impairments such as ON-OFF motor fluctuation, ${ }^{58,65}$ levodopa induced dyskinesias, ${ }^{23}$ and FOG $^{22,71}$ often appear. The motor analysis of standardized MDS-UPDRS III tasks (e.g., forearm pronosupination, heel tapping, gait, and tremor) ${ }^{35,48,60-64,66,69,72-74}$ is another fundamental application that enables clinicians to have objective results about patients' motor performance over time, supporting remote differential diagnosis. Motor performance could also be assessed during $\mathrm{ADL}^{59}$ or by executing virtual games. ${ }^{75}$ Alternative systems involved speech analysis ${ }^{37}$ and facial recognition ${ }^{64}$ as well, which can add useful information about disease onset and progression, as described in MDS-UPDRS tasks 3.1, 3.2. Since sensitive data are acquired, processed, and stored in the cloud, secure data tients, with worsening in their QoL. These people need longterm treatments, therapy adjustments, and monitoring, but often, clinical examinations in hospitals are not sufficient for optimal management of the pathology due to long waiting lists, high traveling distance, working hours lost, etc. The possibility to monitor the patients at home enables the evaluation of many aspects that are not always evident or are infeasible to assess during neurological examinations in clinic, including motor fluctuations and dyskinesias, ${ }^{25-31}$ freezing events, ${ }^{21,71}$ response to therapy adjustments before and after medical intakes, ${ }^{29,43,47}$ and eventually correlated pathologies (e.g., cardiac activities). ${ }^{56}$ Furthermore, the use of a monitoring system in the home environment could eliminate the "white coat effect," which is responsible for better performance in the hospital rather than during daily living activities. $^{44,56}$

Acceptability, ${ }^{32,34,68}$ usability, $^{35,50,62}$ and wearability ${ }^{33}$ are all considerations that require particular attention to have an efficacious system that will actually be used by patients, without affecting their daily activities, both physically, avoiding impairments due to obtrusive heavy devices, and socially, avoiding devices that could be embarrassing and invasive for the users when they are in the community. For these reasons, the use of smartphones ${ }^{52,56}$ or jewelry-like wearable sensors, ${ }^{76,77}$ which are common technological tools, seems to be the best solution to have a portable inexpensive instrument, which is socially accepted and easy to use. Particularly, using internal sensors and algorithms of a smartphone prevents the need for additional hardware, almost for some kind of assessment.

Usability and acceptability from users' perspective are important issues for an operative and effective telemedicine system, as mentioned in several articles included in this review. Nevertheless, just few works reported quantitative results about them. In particular, Ferrari et al. ${ }^{35}$ administered to the users a questionnaire based on a five-point Likert scale for the CUPID system usability and feasibility evaluation. They reported a satisfactory result, with a mean value of 4.5 out of 


\section{ROVINI ET AL.}

5. Similarly, Ginis et al. ${ }^{45}$ also investigated usability for the CUPID system, obtaining very positive responses, as scores on user-friendliness were on average above 4 on a 5-point scale. Ferreira et al., ${ }^{50}$ also proposed to assess the usability of another system, the SENSE-PARK system, that achieved mean score of 2.67 out of 5 on the PSSUQ.

Another matter of debate is the optimal number of sensors to use, because preliminary results suggested that reducing them may lead to loss of potentially relevant information, especially for PD patients, who show a large variability in movements. ${ }^{49}$ However, in accordance with literature, generally less than four sensor devices were used. ${ }^{58}$ Anyway, energy harvesting approaches should be investigated because devices with long battery life are mandatory for long-term monitoring in unconstrained environments. $^{72,73}$

The use of a tele-system for automated assessment of PD symptoms could support the neurologist in remote differential diagnosis, as well as through decision-making support systems. ${ }^{58,65}$ While the test results are automatically uploaded into patient medical records, the system could provide instantaneous feedback to the users, ${ }^{35,52,66}$ allowing the patients to obtain immediate results about their current condition without the direct involvement of any clinics. ${ }^{63}$

Since telehealth systems acquire and manage a wide amount of data, machine learning techniques are needed for their processing, analysis, and aggregation; thus, the results could be appropriately showed to patients and/or clinical staff, through smart user interfaces. ${ }^{22,23}$ Technically, the management of a large amount of data requires attention to data loss and correct transmission of data. ${ }^{25,67,73}$ Ethically, since sensitive data are acquired and processed, adequate measurements for data protection should be applied, including restricted and authenticated access to data, ${ }^{22,60,63}$ secure encrypted data transmission (e.g., SSL, SSH, VPN, and TLS protocols), ${ }^{65,67-69,72}$ and anonymized personal data. ${ }^{59}$

As limitation, most of the articles included in this review involve a limited number of subjects in the experimental sessions and have a lack of randomization, potential recall bias, and likely selection bias. Thus, the clinical validation of the proposed systems cannot be addressed, ${ }^{73}$ and further investigations are required. In addition, sociocultural factors are usually not investigated in these works; therefore, there is a lack of information concerning the influence of relatives on telemedicine services and how gender, education, and working condition could affect their design and provision.

Finally, the development of telehealth systems is a step beyond the simple use of wearable sensors at home, because it means actively including patients and caregivers in the healthcare path, ${ }^{25}$ promoting their empowerment in the management of their health status and disease progression through a conscious involvement. ${ }^{58}$ The concept, indeed, is to transform the patients from end users to the main actors of the healthcare process, favoring the participation and cooperation of patients, caregivers, and clinical staff ${ }^{25}$ to provide the best care available for each patient according to the precision medicine approach. Appropriate training sessions would be organized to enable people to correctly use the system. The possibility to have a more personalized therapy ${ }^{31}$ seems also to increase the feeling of assurance of the patients regarding the appropriate healthcare path to follow. ${ }^{65}$

Generally, the adoption of telemedicine should be accompanied with the transformation of healthcare sector and overcome specific barriers. In terms of organization of the healthcare sector, reimbursement profiles should be defined considering which patients may benefit most and understanding the optimal frequency of telemedicine visits as replacement for in-person encounters. ${ }^{78}$ Furthermore, a uniform regulation is missing in the domain of medical liability, at national and international level, thus hampering the development of telemedicine market in health services. $^{79}$ Telemedicine requires ubiquitous, adequate affordable broadband to support health information exchange to increase access to quality care for all individuals at the right place and the right time when it is needed. ${ }^{78}$

\section{Conclusions}

This review article provides an exhaustive overview of automated systems based on wearable and portable technologies for remote assessment and management of Parkinson's disease. The articles were divided into three categories according to the level of development of the implemented system, considering the automatic evaluation at home of PD symptoms and impairments, the development of mobile applications for PD assessment, and the design of e-Health systems for a complete remote healthcare service. Although they raised limitations, the use of such systems has the potentiality to enhance the PD management and treatment, supporting clinicians in remote monitoring and promoting the active engagement of the patients and their caregivers in the healthcare path. This aims to improve both patients' QoL and clinicians' quality of care toward an optimal personalized therapy.

\section{Acknowledgment}

This work was financially supported by the DAPHNE project (Regione Toscana PAR FAS 2007-2013, Bando FAS Salute 2014, CUP J52I16000170002). 


\section{Authors' Contributions}

ER was responsible for article structure and writing, synthesizing the information from the articles into text and tables. CM was the clinical supervisor, responsible for clinical aspects and contributing in introduction, methodology definition, and search strategies. FC was the scientific supervisor and contributed in methodology definition, article writing, discussion, and conclusion. All authors were involved in article screening and selection. All authors read, provided feedback, and approved the final article.

\section{Author Disclosure Statement}

No competing financial interests exist.

\section{REFERENCES}

1. Olesen J, Gustavsson A, Svensson M, Wittchen HU, Jönsson B. The economic cost of brain disorders in Europe. Eur J Neurol 2012;19: 155-162.

2. Yang J-X, Chen L. Economic Burden Analysis of Parkinson's Disease Patients in China. Parkinsons Dis 2017;2017:1-7.

3. Bovolenta TM, de Azevedo Silva SMC, Arb Saba R, Borges V, Ferraz HB, Felicio AC. Systematic review and critical analysis of cost studies associated with Parkinson's disease. Parkinsons Dis 2017;2017:11.

4. Dorsey ER, Constantinescu R, Thompson JP, et al. Projected number of people with Parkinson disease in the most populous nations, 2005 through 2030. Neurology 2007;68:384-386

5. Fahn S. Clinical aspects of Parkinson disease. In: Nass R, Przedborski S, eds. Parkinson's disease: molecular and therapeutic insights from model systems. 1st Edit. Elsevier, Inc., 2008:3-48.

6. Kadastik-Eerme L, Muldmaa M, Lilles S, Rosenthal M, Taba N, Taba P. Nonmotor features in Parkinson's disease: What are the most important associated factors? Parkinsons Dis 2016;2016:8.

7. Schapira AH, Chauduri RK, Jenner P. Non-motor features of Parkinson disease Nat Rev Neurosci 2017;18:435-450.

8. Martinez-Martin $\mathrm{P}$, Jeukens-Visser $\mathrm{M}$, Lyons $\mathrm{KE}_{\text {, et }}$ al. Health-related quality-of-life scales in Parkinson's disease: Critique and recommendations. Mov Disord 2011;26:2371-2380.

9. Dorsey ER, Vlaanderen FP, Engelen LLPG, et al. Moving Parkinson care to the home. Mov Disord 2016:31:1258-1262.

10. Szewczyk-Krolikowski $K_{1}$ Tomlinson $P$, Nithi $K$, et al. The influence of age and gender on motor and non-motor features of early Parkinson's disease: Initial findings from the Oxford Parkinson Disease Center (OPDC) discovery cohort. Park Relat Disord 2014;20:99-105

11. Vu TC, Nutt JG, Holford NHG. Progression of motor and nonmotor features of Parkinson's disease and their response to treatment. Br J Clin Pharmacol 2012;74:267-283

12. Holford N, Nutt J. Disease progression, drug action and Parkinson's disease: Why time cannot be ignored. Eur J Clin Pharmacol 2008; 64:207-216.

13. Ossig $\mathrm{C}$, Reichmann $\mathrm{H}$. Treatment strategies in early and advanced Parkinson disease. Neurol Clin 2015;33:19-37.

14. Tan L, Jiang T, Tan L, Yu J-T. Toward precision medicine in neurological diseases. Ann Transl Med 2016:4:104.

15. Bu L, Yang $\mathrm{K}$, Xiong $W$, et al. Toward precision medicine in Parkinson's disease. Ann Transl Med 2016;4:26.
16. Chen J, Mullins CD, Novak $P$, Thomas SB. Personalized strategies to activate and empower patients in healthcare and reduce health disparities. Heal Educ Behav 2016:43:25-34.

17. Espay AJ, Bonato $P, N a h a b ~ F B$, et al. Technology in Parkinson's disease: Challenges and opportunities. Mov Disord 2016;31:1272-1282.

18. Wicks P, Stamford J, Grootenhuis MA, Haverman L, Ahmed S. Innovations in e-health. Qual Life Res 2014;23:195-203.

19. Pasluosta CF, Gassner H, Winkler J, Klucken J, Eskofier BM. An emerging era in the management of Parkinson's disease: Wearable technologies and the internet of things. IEEE J Biomed Heal Informatics 2015;19: 1873-1881.

20. Moher D, Shamseer L, Clarke M, et al. Preferred reporting items for systematic review and meta-analysis protocols (PRISMA-P) 2015 statement. Syst Rev 2015;4:1.

21. Rodrıguez-Martin D, Samà A, Perez-López C, et al. Home detection of freezing of gait using Support Vector Machines through a single waist-worn triaxial accelerometer. PLoS One 2017;12:1-26.

22. Mazilu S, Blanke U, Dorfman $M$, et al. A wearable assistant for gait training for Parkinson's Disease with Freezing of Gait in Out-of-the-Lab Environments. ACM Trans Interact Intell Syst 2015;5:1-31.

23. Tzallas AT, Tsipouras MG, Rigas G, et al. PERFORM: A system for monitoring, assessment and management of patients with Parkinson's disease. Sensors 2014;14:21329-21357.

24. Stack $E$, King $R$, Janko $B$, et al. Could in-home sensors surpass human observation of people with Parkinson's at high risk of falling? An ethnographic study. Biomed Res Int 2016;2016:1-10

25. Cancela J, Pastorino M, Arredondo MT, Hurtado O. A telehealth system for Parkinson's disease remote monitoring: The PERFORM approach. Conf Proc IEEE Eng Med Biol Soc 2013;2013:7492-7495.

26. Ramsperger R, Meckler $\mathrm{S}$, Heger $\mathrm{T}$, et al. Continuous leg dyskinesia assessmen in Parkinson's disease -clinical validity and ecological effect. Park Relat Disord 2016;26:41-46

27. Pérez-López C, Samà A, Rodrıguez-Martin D, et al. Dopaminergic-induced dyskinesia assessment based on a single belt-worn accelerometer. Artif Intell Med 2016;67:47-56.

28. Roy SH, Cole BT, Member SSSS, et al. Resolving signal complexities for ambulatory monitoring of notor function in Parkinson's Disease. Conf Proc IEEE Eng Med Biol Soc 2011;2011:4832-4835.

29. Pulliam CL, Heldman DA, Brokaw EB, Mera TO, Mari ZK, Burack MA. Continuous assessment of Levodopa response in Parkinson's Disease using wearable motion sensors. IEEE Trans Biomed Eng 2017;9294:1.

30. Fisher JM, Hammerla NY, Ploetz $T$, Andras P, Rochester L, Walker RW. Unsupervised home monitoring of Parkinson's disease motor symptoms using body-worn accelerometers. Park Relat Disord 2016;33:44-50.

31. Das S, Amoedo B, Torre FD la, Hodgins J. Detecting Parkinson's symptoms in uncontrolled home environments: A multiple instance learning approach. Conf Proc IEEE Eng Med Biol Soc 2012;2012:3688-3691.

32. Fisher JM, Hammerla NY, Rochester L, Andras P, Walker RW. Body-worn sensors in Parkinson's Disease: Evaluating their acceptability to patients. Telemed e-Health 2016;22:63-69.

33. Cancela J, Pastorino M, Arredondo MT, Nikita KS, Villagra F, Pastor MA. Feasibility study of a wearable system based on a wireless body area network for gait assessment in Parkinson's disease patients. Sensors 2014;14 $4618-4633$.

34. Cancela J, Pastorino M, Tzallas A, et al. Wearability assessment of a wearable system for Parkinson's disease remote monitoring based on a body area network of sensors. Sensors 2014;14:17235-17255.

35. Ferrari A, Ginis $P$, Nieuwboer $A$, Greenlaw R, Muddiman A, Chiari L. Handling gait impairments of persons with $P D$ by means of real-time biofeedback in a daily life environment. In: Chang C, Chiari L, Cao Y, Jin H, Mokhtari M, Aloulou 


\section{ROVINI ET AL.}

H, eds. Inclusive Smart Cities and Digital Health (ICOST). Vol 9677. Cham: Springer, 2016:250-261.

36. Wagner A, Fixler N, Resheff YS. A wavelet-based approach to monitoring Parkinson's Disease symptoms. In: Int. Conf. on Acoustics, Speech and Signal Processing (ICASSP). New Orleans, LA, IEEE, 2017.

37. Monteiro A, Dubey H, Mahler L, Yang Q, Mankodiya K. Fit: A Fog computing device for speech tele-treatments. In: Int. Conf. on Smart Computing (SMARTCOMP). St. Louis, MO, IEEE, 2016:10-12.

38. Cook DJ, Schmitter-Edgecombe M, Dawadi P. Analyzing activity behavior and movement in a naturalistic environment using smart home. J Biomed Heal Informatics 2015;19:1882-1892.

39. Del Din S, Godfrey A, Galna B, Lord S, Rochester L. Free-living gait characteristics in ageing and Parkinson's disease: Impact of environment and ambulatory bout length. J Neuroeng Rehabil 2016;13:46.

40. Mancini M, El-Gohary M, Pearson S, et al. Continuous monitoring of turning in Parkinson's disease: Rehabiliation potential. NeuroRehabilitation 2015;37: 3-10.

41. Weiss A, Sharifi S, Plotnik M, van Vugt JPP, Giladi N, Hausdorff JM. Toward automated, at-home assessment of mobility among patients with Parkinson disease, using a body-worn accelerometer. Neurorehabil Neural Repair 2011; 25:810-818.

42. Zampieri C, Salarian A, Carlson-Kuhta P, Nutt JG, Horak FB. Assessing mobility at home in people with early Parkinson's disease using an instrumented Timed Up and Go test. Park Relat Disord 2011;17: 277-280.

43. El-Gohary M, McNames J, Chung K, Aboy M, Salarian A, Horak F. Continuous at-home monitoring of tremor in patients with Parkinson's Disease. Anal Biomed Signals Images 2010:420-424.

44. El-Gohary M, Pearson S, McNames J, et al. Continuous monitoring of turning in patients with movement disability. Sensors 2014;14:356-369.

45. Ginis $P$, Nieuwboer $A$, Dorfman $M$, et al. Feasibility and effects of home-based smartphone-delivered automated feedback training for gait in people with Parkinson's disease: A pilot randomized controlled trial. Park Relat Disord 2016;22:28-34

46. Rodrıguez-Molinero A, Samà A, P'erez-López C et al. Analysis of correlation between an accelerometer-based algorithm for detecting Parkinsonian gait and UPDRS subscales. Front Neurol 2017;8:3-8.

47. Rahimi F, Bee C, Duval C, Boissy P, Edwards R, Jog M. Using ecological whole body kinematics to evaluate effects of medication adjustment in Parkinson disease. J Parkinsons Dis 2014;4:617-627.

48. Patel $\mathrm{S}$, Chen B-R, Mancinelli $\mathrm{C}$, et al. Longitudinal monitoring of patients with Parkinson's disease via wearable sensor technology in the home setting. Conf IEEE Eng Med Biol Soc 2011;2011:1552-1555.

49. Rahimi F, Duval C, Jog M, et al. Capturing whole-body mobility of patients with Parkinson disease using inertial motion sensors: Expected challenges and rewards. Conf IEEE Eng Med Biol Soc 2011;2011: 5833-5838.

50. Ferreira JJ, Godinho C, Santos AT, et al. Quantitative home-based assessment of Parkinson's symptoms: The SENSE-PARK feasibility and usability study. BMC Neurol 2015;15:89.

51. Nikolova S. 28 percent purely digital players transform the mhealth market. Research2Guidance - mHealth app developer economics study 2017. https://research2guidance.com/28-percent-digital-players-aretransforming-the-mhealth-market. Published 2017. (last accessed December 21, 2017).

52. Milosevic M, Jovanov E, Milenković A. Quantifying Timed-Up-and-Go Test: A smartphone implementation. In: 10th Int. Conf. on Body Sensor Networks (BSN). Cambridge, MA, USA, IEEE, 2013:1-6.

53. LeMoyne R, Mastroianni T, Cozza M, Coroian C, Grundfest W. Implementation of an iPhone as a wireless accelerometer for quantifying gait characteristics. Conf IEEE Eng Med Biol Soc 2010;2010:3847-3851.
54. Daneault J-F, Carignan B, Codère CÉ, Sadikot AF, Duval C. Using a smart phone as a standalone platform for detection and monitoring of pathological tremors. Front Hum Neurosci 2013;6:357.

55. Rigas G, Gatsios D, Fotiadis DI, et al. Tremor UPDRS Estimation in home environment. Conf IEEE Eng Med Biol Soc 2016;2016:3642-3645.

56. Richer $\mathrm{R}$, Groh $\mathrm{BH}$, Blank $\mathrm{P}$, et al. Unobtrusive real-time heart rate variability analysis for the detection of orthostatic dysregulation. In: 13th Annual Body Sensor Networks Conference (BSN). San Francisco, CA, USA, IEEE, 2016: 189-193.

57. Huang Y-P, Yang W-J, Lin S-Y. An intelligent approach to identify elderly body information. In: Int. Conf. on Automation Science and Engineering (CASE). Taipei, Taiwan, IEEE; 2014:824-829.

58. Cancela J, Mascato SV, Gatsios D, et al. Monitoring of motor and non-motor symptoms of Parkinson's disease through a mHealth platform. Conf IEEE Eng Med Biol Soc 2016;2016:663-666.

59. Silva de Lima AL, Hahn T, de Vries NM, et al. Large-Scale wearable sensor deployment in Parkinson's patients: The Parkinson@Home Study Protocol. JMIR Res Protoc 2016;5:e172.

60. Dzhagaryan A, Milenkovic A, Jovanov A, Milosevic A. Smart Button: A wearable system for assessing mobility in elderly. In: 17th Int. Conf. on E-Health Networking, Application and Services (HealthCom). Boston, MA, IEEE, 2015: 416-421.

61. Pan D, Dhall R, Lieberman A, Petitti DB. A mobile Cloud-based Parkinson's Disease assessment system for home-based monitoring. JMIR mHealth uHealth 2015;3:e29.

62. Piro NE, Baumann L, Tengler M, Piro L, Blechschmidt-Trapp R. Telemonitoring of patients with Parkinson's disease using inertia sensors. Appl Clin Inform 2014;5:503-511.

63. Sahyoun A, Chehab K, Al-madani O, Aloul F, Sagahyroon A. ParkNosis: Diagnosing Parkinson's Disease using mobile phones. In: 18th Int. Conf. on E-Health Networking, Applications and Services (Healthcom). Munich, Germany, IEEE, 2016:1-6.

64. Sharma V, Mankodiya K, De La Torre F, et al. SPARK: Personalized Parkinson disease interventions through synergy between a smartphone and a smartwatch. Lecture Notes Comp Sci 2014;8519:103-114.

65. Tsiouris KM, Gatsios D, Rigas G, et al. PD_Manager: An mHealth platform for Parkinson's disease patient management. Healthc Technol Lett 2017;4: 102-108.

66. Ferrari $A$, Ginis $P$, Hardegger $M$, Casamassima $F$, Rocchi $L$, Chiari L. A mobile Kalman-filter based solution for the real-time estimation of spatio-temporal gait parameters. IEEE Trans Neural Syst Rehabil Eng 2016; 24:764-773.

67. Chen B-R, Patel S, Buckley T, et al. A Web-based system for home monitoring of patients with Parkinson's disease using wearable sensors. Trans Biomed Eng 2011;58:831-836.

68. Giansanti D, Maccioni G, Morelli S. An experience of health technology assessment in new models of care for subjects with Parkinson's disease by means of a new wearable device. Telemed e-Health 2008;14:467-472.

69. Kostikis N, Arnaoutoglou M, Kotsavasiloglou C. A Smartphone-based tool for assessing Parkinsonian hand tremor. J Biomed Heal Informatics 2015;19: 1835-1842.

70. Lawo M, Herzog 0. Wearable computing for medical applications: Personal health system for Parkinson's disease patients. In: 8th Int. Conf. \& Expo on Emerging Technologies for a Smarter World (CEWIT). Long Island, NY, USA: IEEE; 2011:1-5.

71. Kim $H_{\text {, Lee }} H J$, Lee $W$, et al. Unconstrained detection of Freezing of Gait in Parkinson's disease patients using smartphone. Conf IEEE Eng Med Biol Soc 2015;2015:3751-3754.

72. Patel $S$, Chen B-R, Buckley $T$, et al. Home monitoring of patients with Parkinson's disease via wearable technology and a web-based application. Conf IEEE Eng Med Biol Soc 2010;2010:4411-4414. 
73. Barroso MC, Esteves GP Nunes TP Silva LMG, Faria ACD, Melo PL A telemedicine instrument for remote evaluation of tremor: Design and initial applications in fatigue and patients with Parkinson's Disease. Biomed Eng Online 2011:10:14.

74. Nguyen HT, Vu CC, Phan VQ, Nguyen VD, Nguyen TD. Design system to remotely monitor patients with Parkinson's Disease. 5th Int Conf Biomed Eng Vietnam. 2015;46:104-105.

75. Synnott J, Chen L, Nugent CD, Moore G. WiiPD An approach for the objective home assessment of Parkinson's disease. Conf IEEE Eng Med Biol Soc 2011; 2011:2388-2391.

76. Esposito D, Cavallo F. Preliminary design issues for inertial rings in Ambient Assisted Living applications. In: Conference Record - IEEE Instrumentation and Measurement Technology Conference. Pisa, Italy, IEEE 2015;2015.

77. Butt AH, Rovini E, Esposito D, Rossi G, Maremmani C, Cavallo F. Biomechanical parameter assessment for classification of Parkinson's disease on clinical scale. Int J Distrib Sens Networks 2017;13.

78. InsitituteofMedicine. Challenges in Telehealth. In: The Role of Telehealth in an Evolving Healthcare Environment: Workshop Summary. Washington, DC The National Academies Press; 2012:17-29.
79. Raposo VL. Telemedicine: The legal framework (or the lack of it) in Europe. GMS Health Technol Assess 2016;12: Doc03

Address correspondence to: Filippo Cavallo, PhD

The BioRobotics Institute

Scuola Superiore Sant'Anna

Viale Rinaldo Piaggio 34

Pontedera (PI) 56025

Italy

E-mail: filippo.cavallo@santannapisa.it

Received: February 7, 2018

Revised: March 18, 2018

Accepted: March 22, 2018

AU1: Please identify (highlight or circle) all authors' surnames for accurate indexing citations.

AU2: The Publisher requests for readability that no paragraph exceeds 15 typeset lines. Please check for long paragraphs and divide where needed.

AU3: In Ref. 5, please mention the location of the publisher.

AU4: In Ref. 77, please mention the volume number or page number, whichever is missing.

AU5: In Ref. 78, please mention the editors name.

AU6: Please define PSD.

AU7: Labels $a$ and $b$ are in the legend of Fig. 3, but not found in figure. Please check. 